(C) 1998 International Press

Adv. Theor. Math. Phys. 2 (1998) 601-618

\title{
Gravitational Anomaly Cancellation for $M$-Theory Fivebranes
}

\author{
Dan Freed ${ }^{a, 1}$, Jeffrey A. Harvey ${ }^{b}$, Ruben Minasian ${ }^{c}$, Gregory \\ Moore $^{c}$ \\ ${ }^{a}$ School of Mathematics \\ Institute for Advanced Study \\ Olden Lane \\ Princeton, NJ 08540 \\ ${ }^{b}$ Enrico Fermi Institute \\ 5640 Ellis Avenue \\ University of Chicago \\ Chicago, IL 60637 \\ ${ }^{c}$ Department of Physics \\ Yale University \\ New Haven, CT 06520
}

\begin{abstract}
We study gravitational anomalies for fivebranes in $\mathrm{M}$ theory. We show that an apparent anomaly in diffeomorphisms acting on the normal bundle is cancelled by a careful treatment of the M theory ChernSimons coupling in the presence of fivebranes. One interesting aspect of our treatment is the way in which a magnetic object (the fivebrane) is smoothed out through coupling to gravity and the resulting relation between antisymmetric tensor gauge transformations and diffeomorphisms in the presence of a fivebrane.
\end{abstract}

e-print archive: http://xxx.lanl.gov/abs/hep-th/9803205

${ }^{1}$ Permanent address: Dept. of Mathematics, University of Texas, Austin, TX 78712. 


\section{Introduction}

$\mathrm{M}$ theory is believed to be a consistent theory of quantum gravity which at low energies reduces to the unique supergravity theory in $D=11$ spacetime dimensions. $M$ theory contains two types of extended objects, membranes and fivebranes. Membranes have odd-dimensional worldvolumes and so there are no continous anomalies associated with the zero modes of a membrane. The cancellation of global membrane anomalies has been demonstrated in [1]. Fivebranes on the other hand have even dimensional worldvolumes and chiral zero modes so a computation is needed to see if there are continous anomalies in the presence of fivebranes. We will not investigate global anomalies for fivebranes in this paper.

A fivebrane of $\mathrm{M}$ theory with worldvolume $W_{6}$ embedded into elevendimensional spacetime $M_{11}$ breaks the Lorentz symmetry from $S O(10,1)$ to $S O(5,1) \times S O(5) .{ }^{2}$ If we believe that $\mathrm{M}$ theory is a well defined theory then diffeomorphisms or equivalently local Lorentz transformations which map the fivebrane to itself should be symmetries of the theory.

Diffeomorphisms preserving the fivebrane worldvolume $W_{6} \rightarrow W_{6}$ are generated by vector fields acting either as diffeomorphisms of the fivebrane worldvolume $W_{6}$ or as $S O(5)$ gauge transformations on the connection on the normal bundle. Using the metric the normal bundle may be regarded as a bundle with metric and connection and structure group $S O(5)$. The potential anomalies in worldvolume diffeomorphisms and $S O(5)$ gauge transformations have two obvious sources. The first is the presence of chiral zero modes on the fivebrane worldvolume. For a charge one fivebrane the zero modes consist of a tensor multiplet of $(2,0), D=6$ supersymmetry. The chiral fields in this multiplet consist of a chiral fermion transforming in the spinor representation of $S O(5)$ and a two-form potential with anti-self-dual field strength which is a singlet under $S O(5)$. The anomaly due to these zero modes can be computed from the standard descent formalism [2-5] and is determined by descent on an eight-form $I_{8}^{z m}$. That is, we have $I_{8}=d I_{7}^{(0)}$ and $\delta I_{7}^{(0)}=d I_{6}^{(1)}$ and the anomaly is given by

$$
2 \pi \int_{W_{6}} I_{6}^{z m(1)}
$$

The second source of anomalies comes from the presence in supergravity of a coupling

$$
\Delta S=\int_{M_{11}} C_{3} \wedge I_{8}^{b}(R)
$$

\footnotetext{
${ }^{2}$ Since we will be considering fermions we should really be discussing the covering groups $\operatorname{Spin}(n)$, this distinction will not be important in what follows
} 
with $I_{8}^{b}$ a specific eight-form constructed out of the curvature on $M_{11}$. Integrating by parts and taking the variation of this term gives

$$
\delta \Delta S=\int_{M_{11}} d G_{4} \wedge I_{6}^{b(1)} .
$$

The fivebrane of $M$ theory acts as a magnetic source for the three-form potential $C_{3}$ of $\mathrm{M}$ theory. With $G_{4}=d C_{3}$ the corresponding field strength this means, roughly speaking, that

$$
d G_{4}=2 \pi \delta_{5}
$$

where $\delta_{5}$ is a five-form which integrates to one in the directions transverse to the fivebrane and has delta function support on the fivebrane ${ }^{3}$. We thus have for the total gravitational anomaly

$$
2 \pi \int_{W_{6}}\left(I_{6}^{z m}+I_{6}^{b}\right)^{(1)}=2 \pi \int_{W_{6}}\left(\frac{p_{2}(N)}{24}\right)^{(1)}
$$

with $p_{2}(N)$ the second Pontrjagin class of the normal bundle [6]. The fact that anomalies in diffeomorphisms of the tangent bundle cancel between these two sources was pointed out in [7]. If we believe that $M$ theory exists and that the fivebrane of $M$ theory is a well defined object then there must be some additional mechanism which cancels the anomaly in diffeomorphisms of the normal bundle. The cancellation of the normal bundle anomaly has been investigated in $[8,9,10]$, but a completely satisfactory answer has not yet emerged.

In field theory there are many examples where a smooth soliton solution of the field theory has chiral zero modes with an anomaly which is cancelled by inflow from the bulk [11]. This cancellation is inevitable in field theory since if the original theory was consistent then the effective action must make sense for any background fields including those of a smooth soliton. In theories including gravity the situation is more problematic. The extremal fivebrane of $\mathrm{M}$ theory is non-singular, but becomes singular when perturbed $[12,13]$. In order to study anomalies it is necessary to study not just a particular fivebrane configuration but families of fivebrane configurations.

There are two related ways to understand the need for families of fivebranes. First, the problem of anomalies is the problem of defining the effective action $e^{i S_{e f f}}$ as a function of the fields of the theory. Thus the effective action is a section of a line bundle over field space. The anomaly vanishes if we can trivialize this bundle. Studying this question involves studying families of field configurations. From a local point of view anomalies involve a lack of conservation of a current or of the energy-momentum tensor in the

\footnotetext{
${ }^{3}$ We will soon give a much more precise definition of $\delta_{5}$
} 
presence of background fields. In order to study this conservation we have to turn on gravitational fields in addition to the background fields of the fivebrane. Since the fivebrane becomes singular when we vary the metric it is far from clear that the anomalies must in fact vanish. A direct approach would involve evaluation of the Rarita-Schwinger operator in backgrounds with a horizon and singularity and would be problematic if not impossible.

In this paper we will not try to study the fivebrane directly as a solution of $D=11$ supergravity. Rather we will study the fivebrane as a magnetic source for the three-form potential of $\mathrm{M}$ theory and will divide the fields up into bulk fields and zero mode fields which are localized on the fivebrane. We will show that a careful treatment in this framework allows us to understand the cancellation of all anomalies. We leave to the future the very interesting question of the relation of this approach to that based on a direct study of solutions to supergravity.

We conclude this introduction with a brief comment about the descent formalism. In the physics literature on anomalies one commonly writes Chern-Weil forms such as $I_{8}$ above as differentials of Chern-Simons forms $I_{7}^{(0)}$. This is valid globally if we fix a reference trivial connection, but in general the Chern-Simons forms only exist locally. In the supergravity theory discussed here we do not want to impose unnecessary global restrictions on the spacetime and the fivebrane, so the descent equations are only valid locally. We make some brief comments about the global structure in section 4. A more complete treatment, together with an exposition of the anomaly cancellation in that global framework, will appear in [14].

In this paper we will follow the conventions and normalizations of [6]. In order to suppress many factors of $2 \pi$ in various formulae we define $\phi_{3}=$ $C_{3} / 2 \pi$ and $C_{4}=G_{4} / 2 \pi$ with $C_{3}$ the three form potential of M theory and $G_{4}$ its field strength.

\section{The Fivebrane Source}

Consider a fivebrane of $\mathrm{M}$ theory located at $y^{a}=0, a=1,2, \ldots 5$ and with longitudinal coordinates $x^{\mu}, \mu=0,1, \ldots 5$. The most naive expression for the Bianchi identity in the presence of the fivebrane is

$$
d\left(f_{4}=\delta\left(y^{1}\right) \cdots \delta\left(y^{5}\right) d y^{1} \wedge \cdots \wedge d y^{5} .\right.
$$

The quantity on the right hand side is a five-form with integral one over the transverse space and delta function support on the fivebrane. However as discussed above, we need to consider families of metrics so the above expression could at most be correct locally. While a delta function source is sufficient for computations where $C_{3}$ enters linearly, as in (1.2), we will soon encounter a Chern-Simons term which is cubic in $C_{3}$. In order to have a 
completely well defined and non-singular prescription in such cases we need to smooth out the delta function source. Having done this we will see that in the presence of a non-zero $S O(5)$ connection on the normal bundle we will have to modify the right hand side of (2.1) in order that it transform covariantly under $S O(5)$ gauge transformations.

In order to define the fivebrane more carefully we first use the metric to define a radial direction away from the fivebrane and we cut out a disc of radius $\epsilon$ around the fivebrane. That is, we remove a tubular neighborhood of the fivebrane of radius $\epsilon$. Let $D_{\epsilon}\left(W_{6}\right)$ denote the total space of the resulting disc bundle with base $W_{6}$ and fibers the discs of radius $\epsilon$. We will define all bulk integrals as limits as $\epsilon$ goes to zero of integrals over $M_{11}-D_{\epsilon}\left(W_{6}\right)$ :

$$
\int_{M_{11}} \mathcal{L} \equiv \lim _{\epsilon \rightarrow 0} \int_{M_{11}-D_{\epsilon}\left(W_{6}\right)} \mathcal{L} .
$$

We will later integrate by parts and use the fact the 10-dimensional boundary of $M_{11}-D_{\epsilon}\left(W_{6}\right)$ is the total space of the $S^{4}$ - sphere bundle over $W_{6}$ of radius $\epsilon$, whose total space we denote $S_{\epsilon}\left(W_{6}\right)$.

In order to smooth out the fivebrane source we choose a smooth function of the radial direction with transverse compact support near the fivebrane, $\rho(r)$, with $\rho(r)=-1$ for sufficiently small $r$ and $\rho(r)=0$ for sufficiently large $r$. The bump form $d \rho$ then has integral one in the radial direction. The smoothed form of (2.1) should then read

$$
d C_{4}=d \rho \wedge e_{4} / 2,
$$

where $d e_{4}=0, e_{4}$ is gauge invariant under $S O(5)$ transformations of the normal bundle, $e_{4} / 2$ has integral one over the fibers of $S_{\epsilon}$, and $d \rho \wedge e_{4} / 2$ should reduce to the naive expression on the r.h.s of (2.1) for a flat infinite fivebrane when $d \rho$ approaches a delta function. Physically what we are doing is smoothing out the magnetic charge of the fivebrane to a sphere of magnetic charge linking the horizon.

The construction of the smoothed out source involves standard mathematics [15]. The right hand side of (2.3) involves differential forms which arise in a geometric construction of the Thom class of an oriented vector bundle, in this case the normal bundle to $W_{6}$ in $M_{11}$. As described in [15] we may identify the total space of the normal bundle with a tubular neighborhood of $W_{6}$ in $M_{11}$. With this identification the differential form $d \rho \wedge e_{4} / 2$ represents the Thom class of the normal bundle and $e_{4} / 2$ is the global angular form. Although the properties of $e_{4}$ follow from general principles, an explicit local formula is useful for constructing explicit objects which will later appear in the $\mathrm{M}$ theory action.

We have $E \rightarrow W_{6}$ a rank 5 real vector bundle with metric and connection. Let $P \rightarrow W_{6}$ be the principal $S O(5)$ bundle associated to the rank 5 bundle 
E. Following [16] we work on $P \times S^{4}$ and construct a basic form which descends to the sphere bundle $S(E)$. Think of $S^{4} \subset \mathbb{R}^{5}$, choose coordinates $y^{a}$ for $\mathbb{R}^{5}$ and let $\hat{y}^{a} \equiv y^{a} / r$. Of course, $\hat{y}^{a}$ is defined only outside of $0 \in$ $\mathbb{R}^{5}$, which corresponds to the complement of the zero section of $E$. On that complement the pullback of $E$ has a tautological line subbundle, and a perpendicular oriented 4-plane bundle which we call $F$. Readers with less tolerance for mathematics can pick a gauge and with little harm done simply think of the $\hat{y}^{a}$ as isotropic coordinates on the $S^{4}$ fibres of $S(E)$.

The $S O(5)$ bundle is equipped with a globally defined connection $\Theta^{a b}=$ $-\Theta^{b a}$. (We identify so(5) $\cong \Lambda^{2} \mathbb{R}^{5}$.) The Lie algebra so(5) acts on $P \times S^{4}$ in the standard way and we have horizontal forms:

$$
\begin{aligned}
(D \hat{y})^{a} & \equiv d \hat{y}^{a}-\Theta^{a b} \hat{y}^{b} \\
F^{a b} & =d \Theta^{a b}-\Theta^{a c} \wedge \Theta^{c b} .
\end{aligned}
$$

We now consider the forms:

$$
\begin{aligned}
& \epsilon_{a_{1} \cdots a_{5}}(D \hat{y})^{a_{1}} \cdots(D \hat{y})^{a_{4}} \hat{y}^{a_{5}} \\
& \epsilon_{a_{1} \cdots a_{5}} F^{a_{1} a_{2}} \wedge(D \hat{y})^{a_{3}}(D \hat{y})^{a_{4}} \hat{y}^{a_{5}}
\end{aligned}
$$

and

$$
\epsilon_{a_{1} \cdots a_{5}} F^{a_{1} a_{2}} \wedge F^{a_{3} a_{4}} \hat{y}^{a_{5}}
$$

These forms are all annihilated by $\iota(X), \mathcal{L}(X)$, for $X \in \mathbf{s o}(\mathbf{5})$, where $\iota(X)$ is the contraction and $\mathcal{L}(X)$ is the Lie derivative with respect to the vector field $X$. It follows that these forms are basic and descend to $S(E)$. Moreover, (2.5) restricts to the volume form on the $S^{4}$ fiber and thus reduces to the naive expression in (2.1) in the appropriate limit. However, (2.5) is not closed. One can use the identities

$$
\begin{aligned}
d \hat{y}^{a} & =\Theta^{a b} \hat{y}^{b}+(D \hat{y})^{a} \\
d(D \hat{y})^{a} & =\Theta^{a b}(D \hat{y})^{b}-F^{a b} \hat{y}^{b} \\
d F^{a b} & =-F^{a c} \Theta^{c b}+F^{b c} \Theta^{c a}
\end{aligned}
$$

plus the rotational invariance of the forms and the fact that $\hat{y}^{a}(D \hat{y})^{a}=0$ to show that up to an overall scale there is a unique closed linear combination of (2.5) - (2.7).

Equivalently, the curvature of the oriented 4-plane bundle $F$ defined above is the restriction to $F$ of the curvature of $E$ minus a second fundamental form term. The Pfaffian of this curvature is represented by the basic 
4-form

$$
\begin{aligned}
& e_{4}(\Theta)=\frac{1}{64 \pi^{2}}\left(\epsilon_{a_{1} \cdots a_{5}}(D \hat{y})^{a_{1}}(D \hat{y})^{a_{2}}(D \hat{y})^{a_{3}}(D \hat{y})^{a_{4}} \hat{y}^{a_{5}}\right. \\
& \left.-2 \epsilon_{a_{1} \cdots a_{5}} F^{a_{1} a_{2}} \wedge(D \hat{y})^{a_{3}} \wedge(D \hat{y})^{a_{4}} \hat{y}^{a_{5}}+\epsilon_{a_{1} \cdots a_{5}} F^{a_{1} a_{2}} \wedge F^{a_{3} a_{4}} \hat{y}^{a_{5}}\right)
\end{aligned}
$$

One can apply the standard descent formalism to expressions of the form (2.9). For example, assuming that the normal bundle is trivial and choosing $\Theta=0$ as a basepoint reference connection we have

$$
\begin{aligned}
e_{3}^{(0)}(\Theta, \hat{y})=\frac{1}{32 \pi^{2}} \epsilon_{a_{1} \cdots a_{5}} & \left(\Theta^{a_{1} a_{2}} d \Theta^{a_{3} a_{4}} \hat{y}^{a_{5}}\right. \\
& \left.-\frac{1}{2} \Theta^{a_{1} a_{2}} \Theta^{a_{3} a_{4}} d \hat{y}^{a_{5}}-2 \Theta^{a_{1} a_{2}} d \hat{y}^{a_{3}} d \hat{y}^{a_{4}} \hat{y}^{a_{5}}\right) .
\end{aligned}
$$

More generally one can write such formulae for the difference of two ChernSimons forms for two connections $\Theta_{1}, \Theta_{2}$ on $E$.

The gauge transformations $\delta \Theta^{a_{1} a_{2}}=(D \varepsilon)^{a_{1} a_{2}}$ and $\delta \hat{y}^{a}=\varepsilon^{a a^{\prime}} \hat{y}^{a^{\prime}}$ give

$$
e_{2}^{(1)}(\varepsilon, \Theta, \hat{y})=\frac{1}{16 \pi^{2}} \epsilon_{a_{1} \cdots a_{5}}\left(\varepsilon^{a_{1} a_{2}} d \hat{y}^{a_{3}} d \hat{y}^{a_{4}} \hat{y}^{a_{5}}-\varepsilon^{a_{1} a_{2}} \Theta^{a_{3} a_{4}} d \hat{y}^{a_{5}}\right) .
$$

The above expressions have natural generalizations to all real oriented bundles of odd rank. We give the general formulae in the appendix.

While we do not see a direct connection between the analysis presented here and the discussion in [6] concerning the normal bundle anomaly, it is interesting to note that the last term in (2.9) is very close to the expressions which appear in the discussion there. We expect that upon dimensional reduction our mechanism becomes equivalent to the anomaly cancellation mechanism for the IIA fivebrane described in [6], but we have not worked out the details of this.

\section{Connection to Anomalies}

In giving a precise definition of the fivebrane source we encountered the global angular form $e_{4} / 2$. It is clear from (2.9) that the global angular form depends on the connection on the normal bundle and is closed and gauge invariant under $S O(5)$ gauge transformations acting on the normal bundle. As described above we can thus apply descent:

$$
e_{4}=d e_{3}^{(0)}, \quad \delta e_{3}^{(0)}=d e_{2}^{(1)} .
$$


We will now express the uncanceled anomaly (1.5) in terms of (3.1) using a result of Bott and Cattaneo [16]. Consider a real vector bundle $N \rightarrow M$ of odd rank $2 n+1$, and for convenience fix a metric. Let $\pi$ : $S(N) \rightarrow M$ denote the unit sphere bundle in $N$. Then the lift $\pi^{*} N$ has a tautological line subbundle $L$, and in [16] it is shown that the Euler class of the orthogonal complement $L^{\perp}$ satisfies $\pi_{*}\left[e_{2 n}\left(L^{\perp}\right)^{3}\right]=2 p_{n}(N)$. The factor of 2 is the Euler characteristic of the even dimensional sphere. At the level of cohomology, this formula follows from a simple argument using the splitting principle. If $N$ has an orthogonal connection, then we represent real characteristic classes as differential forms using Chern-Weil representatives. The formula also holds at the level of differential forms, since both sides are gauge invariant and depend on only a finite number of derivatives of the connection. Applying the result of [16] to our case, and applying the descent formalism we have:

$$
\frac{1}{6} \int_{S_{\epsilon}\left(W_{6}\right)} \frac{e_{4}}{2} \wedge \frac{e_{4}}{2} \wedge \frac{e_{2}^{(1)}}{2}=\int_{W_{6}} \frac{\left(p_{2}(N)\right)^{(1)}}{24} .
$$

The $D=11$ supergravity which describes the low-energy limit of $\mathrm{M}$ theory contains a Chern-Simons term

$$
S_{C S}=-\frac{2 \pi}{6} \int_{M_{11}} \not_{3} \wedge d \not_{3} \wedge d \not_{3}^{4}=\frac{2 \pi}{6} \int_{M_{12}} d \not_{3} \wedge d \not \phi_{3} \wedge d \not \zeta_{3},
$$

where $M_{12}$ is a twelve-manifold with boundary $M_{11}$. In the absence of fivebranes we have $G_{4}=d C_{3}$ and $d G_{4}=0$. In the presence of fivebranes we have argued above that this equation should be modified to $d G_{4}=d \rho \wedge e_{4} / 2$. This requires that we modify the relation between $G_{4}$ and $C_{3}$. The modified Bianchi identity is satisfied with

$$
C_{4}=d C_{3}+A \rho e_{4} / 2-B d \rho \wedge e_{3}^{(0)} / 2
$$

where locally $C_{3}$ can be viewed as a small fluctuation field about the fivebrane $^{4}$ and $A+B=1$. Since have smoothed out the fivebrane source we expect on physical grounds that $C_{3}$ and $G_{4}$ should be smooth on the fivebrane, and in fact in the treatment of [6] it is important that $C_{3}$ be well defined on the fivebrane. Since $\rho e_{4}$ is singular at the fivebrane this requires that we take $A=0$ and hence $B=1$.

We thus have

$$
C_{4}=d \not_{3}-d \rho \wedge e_{3}^{(0)} / 2 \text {. }
$$

This relation is quite analogous to the relation $H_{3}=d B_{2}-\omega_{3}$ which occurs in $D=10, N=1$ supergravity coupled to gauge theory and is central to the

\footnotetext{
${ }^{4}$ The global definition of $C_{3}$ is given in the following section.
} 
Green-Schwarz anomaly cancellation mechanism. In particular, the relation (3.5) implies that $C_{3}$ must have an anomalous variation under $S O(5)$ gauge transformations in order that $G_{4}$ be gauge invariant,

$$
\delta \not \zeta_{3}=-d \rho \wedge e_{2}^{(1)} / 2 .
$$

Given the modified relation between $G_{4}$ and $d C_{3}$ we must ask how the Chern-Simons term should be modified. These modifications will involve higher derivative metric interactions. Actually, describing these terms as higher derivative terms is slightly misleading since it presupposes a local description of the physics. However $\sigma_{3}$ is not local in the metric, since we use the exponential map to transfer forms from the total space of the normal bundle to a neighborhood of the fivebrane. We expect that an eventual microscopic derivation will explain this nonlocality or replace it with a local description. With this caveat in mind, there are many higher derivative terms one could add to the supergravity action. These are constrained by physical principles such as supersymmetry and gauge invariance. Here we will only examine the constraints of gauge invariance under diffeomorphism and 3 -form gauge transformations. We introduce the expression $\sigma_{3}$ defined by:

$$
\left(G_{4}-\rho e_{4} / 2=d\left(\phi_{3}-\rho e_{3}^{(0)} / 2\right) \equiv d\left(\phi_{3}-\sigma_{3}\right) .\right.
$$

(Note that $G_{4}$ is not exact.) A natural set of higher order terms relevant to the anomaly cancellation problem is obtained by replacing $C_{3}$ by $\sigma_{3}$ or $d C_{3}$ by $G_{4}, \rho e_{4}$, or $d \sigma_{3}$. One finds in this way twelve linearly independent higher derivative metric interactions. One combination of higher derivative terms which maintains the Chern-Simons structure of the original interaction is

$$
S_{C S}^{\prime}=\lim _{\epsilon \rightarrow 0}-\frac{2 \pi}{6} \int_{M_{11}-D_{\epsilon}\left(W_{6}\right)}\left(\not_{3}-\sigma_{3}\right) \wedge d\left(\phi_{3}-\sigma_{3}\right) \wedge d\left(\not_{3}^{\prime}-\sigma_{3}\right)
$$

and we will take (3.8) as the modified Chern-Simons term. It includes higher derivative interactions involving up to eleven derivatives of the metric. Moreover, it is not gauge invariant by itself under diffeomorphisms. Under diffeomorphisms $(S O(5)$-gauge transformations of the normal bundle) the variation of $C_{3}$ leads to a variation of (3.8). Indeed, it follows from (3.6) that

$$
\delta\left(\not_{3}-\sigma_{3}\right)=-d\left(\rho e_{2}^{(1)} / 2\right)
$$

Computing the variation we have

$$
\delta S_{C S}^{\prime}=\lim _{\epsilon \rightarrow 0} \frac{2 \pi}{6} \int_{M_{11}-D_{\epsilon}\left(W_{6}\right)} d\left(\rho e_{2}^{(1)} / 2\right) \wedge d\left(\phi_{3}^{h}-\sigma_{3}\right) \wedge d\left(\phi_{3}-\sigma_{3}\right)
$$


Integrating by parts and taking the limit and using the fact that $G_{4}$ and $C_{3}$ are smooth near the fivebrane we obtain

$$
\delta S_{C S}^{\prime}=-\frac{2 \pi}{6} \int_{S_{\epsilon}\left(W_{6}\right)} \frac{e_{4}}{2} \wedge \frac{e_{4}}{2} \wedge \frac{e_{2}^{(1)}}{2},
$$

which by (3.2) cancels the remaining anomaly in diffeomorphisms of the normal bundle. In [6] the cancellation of antisymmetric tensor gauge transformation of $C_{3}$ was also studied. It is not hard to see that the modification we have made to the Chern-Simons coupling preserves the cancellation found in $[6]$.

\section{Global Structure}

Far from the fivebrane $d \rho$ vanishes and we have locally $G_{4}=d C_{3}$. On the other hand we have by the definition of a fivebrane that

$$
\int_{S_{\infty}^{4}} q_{4}^{4}=1
$$

so $C_{3}$ cannot be globally well defined. Rather, we must define $C_{3}^{i}$ in patches of an open cover $\mathcal{U}_{i}$ and relate the $C_{3}^{i}$ across patches by antisymmetric tensor gauge transformations, $C_{3}^{i}-C_{3}^{j}=d \Lambda^{i j}$. The appropriate machinery for this construction is the Čech de Rham complex. A very readable account aimed towards physicists can be found in [17]. The situation here is more complicated due to the mixture of tensor gauge transformations and diffeomorphisms required by the Green-Schwarz like structure. Because of the way we have smoothed out the fivebrane source the quantity with constant linking number through an $S^{4}$ surrouriding the fivebrane is $q_{4}^{4}-\rho e_{4} / 2$. In particular

$$
\int_{S_{r}^{4}} q_{4}
$$

varies from 1 to zero as $r$ decreases from large $r$ to small $r$. To explain the consequences for $C_{3}$ we first describe the patching conditions on $C_{3}$ near the fivebrane. Then we summarize briefly a global description; see [14] for a more leisurely exposition.

Choose an open cover $\mathcal{V}_{\alpha}$ for the fivebrane $W_{6}$. Then in the transverse space which we take to be $\mathbb{R}^{5}$ for simplicity we choose radial coordinates and split $S^{4}=S_{+}^{4} \cup S_{-}^{4}$ into northern and southern hemispheres.

We then have patches

$$
\begin{aligned}
& \mathcal{V}_{\alpha} \times S_{+}^{4} \times[r>0] \\
& \mathcal{V}_{\alpha} \times S_{-}^{4} \times[r>0]
\end{aligned}
$$


The antiderivative $e_{3}^{(0)}(\Theta, \hat{y})$ transforms across the overlap region $S^{3} \times I \cong$ $S_{+}^{4} \cap S_{-}^{4}$ by an $S O(5)$ gauge transformation $g_{ \pm}$.

Using the fact that $C_{3}$ is well defined on $W_{6}$ and the gauge invariance of $G_{4}$ we therefore take:

$$
\not_{3}^{+}-\not_{3}^{--}=\frac{1}{2}(1+\rho) d e_{2}^{(1)}\left(g_{ \pm}, \Theta_{+}, \hat{y}_{+}\right)
$$

where $e_{2}^{(1)}$ is the integrated form of the cocycle $e_{2}^{(1)}$ given in (2.11). Note that this is not a an antisymmetric tensor gauge transformation. But we can use the fact that $C_{3}$ has picked up a diffeomorphism variation to write this as:

$$
\begin{aligned}
\mathscr{C}_{3}^{+}-\not_{3}^{-} & =d\left[\frac{(1+\rho)}{2} e_{(2)}^{1}\left(g_{ \pm}, \Theta_{+}, \hat{y}_{+}\right)\right] \\
& -\frac{1}{2} d \rho \wedge e_{(2)}^{1}\left(g_{ \pm}, \Theta_{+}, \hat{y}_{+}\right)
\end{aligned}
$$

which is a sum of an antisymmetric tensor gauge transformation and a diffeomorphism gauge transformation. We thus find that $C_{3}$ is well defined near $W_{6}$. As we move away from the fivebrane $C_{3}$ requires non-trivial transition functions between patches which are a combination of antisymmetric tensor gauge transformations and diffeomorphisms. At infinity these reduce to pure antisymmetric tensor gauge transformations.

A global discussion may be framed in terms of a general " $\Gamma$-calculus," which is an extension of the usual calculus of differential forms. We describe it in terms of an open cover $\left\{U_{i}\right\}$ of $M$ which is good in the sense that all intersections

$$
U_{i_{0} \ldots i_{p}}=U_{i_{0}} \cap \cdots \cap U_{i_{p}}
$$

are contractible. The set of good covers is contractible in a suitable sense, so the particular choice of good cover does not affect the result of any computation. Let $\left(\Gamma^{\bullet}(M), D\right)$ be the total complex of the modified Čech-de Rham complex 


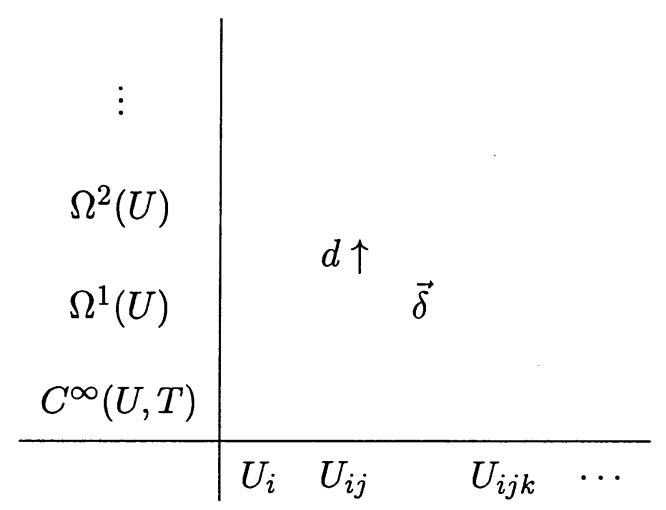

Here $\delta$ is the usual Čech differential, the columns form the de Rham complex modified by replacing 0 -forms by circle-valued functions, and $D=\delta \pm d$ is the total differential. There is a subspace $\Theta^{p}(M) \subset \Gamma^{p}(M)$ of "connection-like" elements:

$$
\Theta^{p}(M)=\left\{\omega \in \Gamma^{p}(M): D \omega \in \Omega^{p+1}(M)\right\} .
$$

For example, an element $\omega=\left\{g_{i j}, \alpha_{i}\right\} \in \Theta^{1}(M)$ has the form

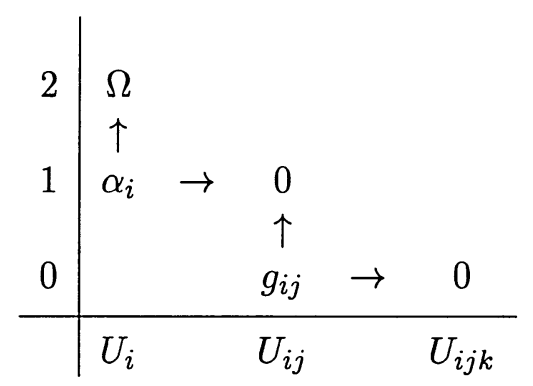

It represents a circle bundle with connection: $g_{i j}$ are the transition functions of some local trivializations, $\alpha_{i}$ are the local connection forms, and $\Omega=D \omega$ is the curvature. Intuitively, $\omega \in \Theta^{p}(M)$ for $p>1$ is a higher degree version of a connection on a circle bundle. An element $\sigma \in \Gamma^{p-1}(M)$ is a trivialization of the trivial bundle $D \sigma \in \Theta^{p}(M)$.

Given the fivebrane $W_{6} \subset M_{11}$ we first define a Poincaré dual form

$$
\Omega(g) \in \Omega^{5}(M)
$$

by the right hand side of (2.3). It depends "functorially" on the metric $g$ on $M$ : the construction is invariant under diffeomorphisms. There is also a diffeomorphism-invariant antiderivative

$$
\mu(g) \in \Omega^{4}(M \backslash W)
$$


on the complement of the fivebrane; it was denoted " $\rho e_{4} / 2$ " previously. By working universally we can also choose a diffeomorphism-invariant connection-like object

$$
\omega(g) \in \Theta^{4}(M)
$$

which is an antiderivative globally: it has curvature $D \omega=\Omega$. Furthermore, there is a trivialization

$$
\sigma(g) \in \Gamma^{3}(M \backslash W)
$$

off of the fivebrane with "covariant derivative" $\mu(g)$ : we write $D \sigma=\mu-$ $\omega$. The quartet $(\omega, \sigma, \Omega, \mu)$ is our global description of the smeared-out fivebrane. It depends on the metric $g$ and the fixed cutoff function $\rho$.

In the absence of any fivebranes the 3 -form field $\phi$ is globally an element of $\Theta^{3}(M)$ with curvature $\mathbb{4}_{4}=D \phi_{3}$. In the presence of the fivebrane, $\phi^{4}$ is a global trivialization

$$
\phi_{3} \in \Gamma^{3}(M)
$$

of $\omega(g)$ with covariant derivative

$$
C_{4} \in \Omega^{4}(M),
$$

i.e., $D \not_{3}=\not_{4}-\omega(g)$. With these definitions the modified Chern-Simons term (3.8) makes sense globally and leads to the anomaly cancellation computed above.

\section{Reduction on a Calabi-Yau 3-fold}

One of the closest relatives of $M$-theory is $\mathcal{N}=1$ supergravity in five dimensions. This theory has Chern-Simons interactions and chiral strings, which can lead to both gauge and gravitational anomalies [18]. A natural way to produce such models is via compactification of $M$-theory on a CalabiYau manifold $X$. In this case there are $h^{1,1}(X)$ independent vector fields, including one graviphoton and $h^{1,1}(X)-1$ vectormultiplets.

Suppose a 5 -brane $W$ wraps a four-cycle $P$ with homology class $[P]=$ $p^{A}\left[\Sigma_{A}\right]$, where $\left[\Sigma_{A}\right]$ is an integral basis for $H_{4}(X, \mathbb{Z})$. At long distances the noncompact part of $W$ is a chiral string in the $\mathbb{R}^{1,4}$ supergravity. The number of left and right-moving bosonic zero modes $N_{L, R}^{B}$ and right-moving fermionic zero modes $N_{R}^{F}$ on the string are given by [19]:

$$
\begin{aligned}
N_{L}^{B} & =6 D+c_{2} \cdot P \\
N_{R}^{B}+\frac{1}{2} N_{R}^{F} & =6 D+\frac{1}{2} c_{2} \cdot P
\end{aligned}
$$


where

$$
c_{2} \cdot P=\int_{P} c_{2}(T X)
$$

and the self-intersection is given by

$$
D=\frac{1}{6} \int_{X} \hat{P}^{3}=D_{A B C} p^{A} p^{B} p^{C},
$$

where $\hat{P}$ is the Poincaré dual class in $H^{2}(X, Z)$.

The BPS string is magnetically charged under the gauge field obtained from the Kaluza-Klein reduction of $C_{3}$ on $X$ dual to $P$, namely, $C_{3}=C_{1} \wedge$ $p^{A} \theta_{A}$ where $C_{1} \in \Omega^{1}\left(\mathbb{R}^{1,4}\right)$ and $\theta_{A}$ is an integral basis of harmonic two-forms on $X$. Defining the field strength associated to $C_{1}$ in the presence of a string requires a treatment very similar to what we presented in the fivebrane case. In particular, the normal bundle is now an $S O(3)$ bundle with connection $\Theta$ and smoothing out the string source requires that we modify the relation between $G_{2}$ and $d C_{1}$ to

$$
d \not_{1}^{\prime}=\not \ell_{2}+d \rho \wedge e_{1}^{(0)}(\Theta) / 2 .
$$

The zero mode spectrum is anomalous and the anomaly is given by descent from

$$
\widetilde{I}_{4}(T W, N)=\frac{1}{48}\left(c_{2} \cdot P\left(p_{1}(T W)+p_{1}(N)\right)+12 D p_{1}(N)\right) .
$$

The cancellation of anomalies in diffeomorphisms of the tangent bundle by inflow requires a bulk coupling

$$
\Delta S^{5}=\int_{M_{5}-D_{\epsilon}(W)} C_{1} \wedge I_{4}(T M)
$$

where now $I_{4}(T M)=\frac{1}{48}\left(c_{2} \cdot P\right) p_{1}(T M)$. This coupling can be obtained by reduction on $X$ of the eleven-dimensional bulk term (1.2). It is easy to see that

$$
\iota^{*}\left(I_{4}(T M)\right)=\widetilde{I}_{4}(T W, N)-\frac{D}{4} p_{1}(N),
$$

where $\iota$ is the inclusion map. Again there is a part of the anomaly involving gauge transformations of the normal bundle which is uncancelled by the inflow. It is interesting to note that the anomaly of the normal bundle arises only when the self-intersection is non-zero which is precisely the condition for having a Chern-Simons interaction in five dimensions. Thus the mechanism 
for cancellation of the normal bundle anomaly should be the same as in $M$ theory.

The modified Chern-Simons coupling is

$$
S^{\prime}{ }_{C S}^{5}=\lim _{\epsilon \rightarrow 0}-12 D \pi \int_{M_{5}-D_{\epsilon}(W)}\left(\phi_{1}-\sigma_{1}\right) \wedge d\left(\phi_{1}-\sigma_{1}\right) \wedge d\left(\phi_{1}-\sigma_{1}\right) .
$$

The anomaly in pure $C_{1}$ gauge transformations is compensated by a phase factor coming from the coupling of $C_{1}$ to the string worldsheet in a way completely analogous to the discussion of $C_{3}$ antisymmetric tensor gauge transformations in [6].

The cancellation of the anomaly in gauge transformations of the normal bundle follows as in the fivebrane case upon application of the relevant version of the Bott-Catteneo formula:

$$
\int_{S_{\epsilon}\left(W_{2}\right)} e_{2} \wedge e_{2} \wedge e_{0}^{(1)}=2 \int_{W_{2}}\left(p_{1}(N)\right)^{(1)}
$$

It would be interesting to explore whether there are implications of this discussion for the black hole entropy following the discussion in [19].

\section{Acknowledgments}

We would like to thank S. Shatashvili for participation in the beginning of this project and for many discussions on the fivebrane anomaly in $\mathrm{M}$ theory. We would also like to thank R. Bott and E. Witten for essential discussions and G. Horowitz for discussions on fivebrane geometry. We also would like to thank the Institute for Theoretical Physics at Santa Barbara for the stimulating workshop which made this collaboration possible. Our joint work there was supported in part by the National Science Foundation under Grant No. PHY94-07194. The work of JH is supported by NSF Grant No. PHY 9600697, RM and GM are supported by DOE grant DE-FG0292ER40704. DF is supported by NSF grants DMS-962698, DMS-9304580, and DMS-9627351 and by the Harmon Duncombe Foundation.

\section{Appendix A: Volume form for all odd rank bundles}

The formulae used in the text for $e_{4}, e_{3}^{(0)}$ etc. have natural extensions to the $S O(2 n+1)$ case. The global angular form can be given, as above, and as in [16] as a basic form on $P \times S^{2 n}$. We find:

$$
e_{2 n}=\frac{1}{2(4 \pi)^{n} n !} \sum_{j=0}^{n}(-1)^{j} \frac{n !}{j !(n-j) !} \epsilon(F)^{j}(D \hat{y})^{2 n-2 j} \hat{y}
$$


where

$$
\epsilon(F)^{j}(D \hat{y})^{2 n-2 j} \hat{y} \equiv \epsilon_{a_{1} \cdots a_{2 n+1}} F^{a_{1} a_{2}} \cdots F^{a_{2 j-1} a_{2 j}}(D \hat{y})^{a_{2 j+1}} \cdots(D \hat{y})^{a_{2 n}} \hat{y}^{a_{2 n+1}}
$$

and the normalization is fixed by noting that the volume form defined by $d^{2 n+1} y=r^{2 n} d r \Omega_{2 n}$ has $\int_{S^{2 n}} \Omega_{2 n}=2 \pi^{n+1 / 2} / \Gamma(n+1 / 2)$.

Similarly, the Chern-Simons form for the general $S O(2 n+1)$ case is given by:

$$
\begin{aligned}
e_{2 n-1}^{0}\left(\Theta_{1}\right) & -e_{2 n-1}^{0}\left(\Theta_{0}\right) \\
& =-\frac{\epsilon}{2(4 \pi)^{n} n !} \int_{0}^{1} d t \sum_{j=0}^{n-1}(-1)^{j} \frac{n !}{j !(n-j-1) !} \Theta\left(F_{t}\right)^{j}\left(D_{t} \hat{y}\right)^{2 n-2 j-2} \hat{y}
\end{aligned}
$$

where $\Theta_{t}=t \Theta_{1}+(1-t) \Theta_{0}$, and $F_{t}=d \Theta_{t}-\Theta_{t}^{2}$ and $D_{t}=\left(d-\Theta_{t}\right)$. The equations simplify for the case that the normal bundle is topologically trivial. In that case there is a canonical choice of basepoint connection $\Theta=0$ for which we may take an antiderivative of the volume form of the sphere $S^{2 n}$.

It is also worth noting that one can give $e_{2 n}$ a Mathai-Quillen-like representation. We can introduce $2 n+1$ orthonormal antighost zeromodes and write, up to a constant, the angular form for the odd rank case as:

$$
e_{2 n}(g)=\frac{1}{2(2 \pi)^{n}} \int \prod_{a=1}^{2 n+1} d \rho^{a} \exp \left[\rho^{a} \rho^{b} F^{a b}-(D \hat{y})^{a} \rho^{a}+\rho^{a} \hat{y}^{a}\right] .
$$

The MQ representative of a rapid-decrease Thom class of odd-rank bundles is therefore:

$$
\Phi^{\text {universal }}=\kappa_{n} e^{-(y, y)} \int^{2 n+1} \prod_{a=1}^{d \rho^{a}} \frac{\sqrt{2 \pi}}{\sqrt{2}} \exp \left[\rho^{a} \rho^{b} \phi^{a b}-(D y)^{a} \rho^{a}+\rho^{a} y^{a}\right],
$$

where $\kappa_{n}$ is a normalization constant and $\phi$ is in the Weil algebra. The expression (A.14) closely resembles the Mathai-Quillen representative of the universal Thom form of even rank bundles, which is a starting point for the development of topological field theory (see, for example, [20]). It would be interesting to see if the above expressions could also be used to develop new topological field theories.

\section{References}

[1] E. Witten, J. Geom. Phys. 22 (1997) 1. 
[2] M.F. Atiyah and I.M. Singer, Proc. Natl. Acad. Sci. 81 (1984) 2597.

[3] L. Faddeev and S. Shatashvili, Theor. Math. Fiz. 60 (1984) 206, English translation in Theor. Math. Phys. 60 (1984) 770.

[4] B. Zumino, "Chiral Anomalies and Differential Geometry", in "Relativity, Groups, and Topology II", Proceedings of the Les Houches summer school, B. S. DeWitt and R. Stora, eds., North-Holland 1984.

[5] For reviews see "Symposium on Anomalies, Geometry, and Topology" W. A. Bardeen and A. R. White, eds., World Scientific 1985; L. AlvarezGaumé and P. Ginsparg, Ann. Phys. 161 (1985) 423.

[6] E. Witten, J. Geom. Phys. 22 (1997) 103.

[7] M. J. Duff, J. T. Liu, and R. Minasian, Nucl. Phys. B452 (1995) 261.

[8] S. P. de Alwis, Phys. Rev. D56 (1997) 7963.

[9] L. Bonora, C. S. Chu, and M. Rinaldi, J. High Energy Phys. 12 (1997) 7; "Anomalies and Locality in Field Theories and M theory", hepth/9712205.

[10] M. Henningson, Nucl. Phys. B515 (1998) 233.

[11] C. G. Callan and J. A. Harvey, Nucl. Phys. B250 (1985) 427.

[12] G. W. Gibbons, G. T. Horowitz, and P. K. Townsend, Class. Quant. Grav. 12 (1995) 297.

[13] G. T. Horowitz and S. F. Ross, J. High Energy Phys. 4 (1998) 15.

[14] D. Freed, "Anomalies, $p$-forms, and M-Theory", in preparation.

[15] R. Bott and L. W. Tu, "Differential Forms in Algebraic Topology", Springer-Verlag, New York 1982.

[16] R. Bott and A. S. Cattaneo, "Integral Invariants of 3-Manifolds", dgga/9710001.

[17] O. Alvarez, Comm. Math. Phys. 100 (1985) 279.

[18] S. Ferrara, R.R. Khuri, and R. Minasian, Phys. Lett. B375 (1996) 81.

[19] J. Maldacena, A. Strominger, and E. Witten, J. High Energy Phys. 12 (1997) 2. 
[20] S. Cordes, G. Moore, and S. Ramgoolam, "Lectures on 2D Yang-Mills Theory, Equivariant Cohomology, and Topological Field Theories", Lectures presented at the 1994 Les Houches Summer School "Fluctuating Geometries in Statistical Mechanics and Field Theory", in Nucl. Phys. Proc. Suppl. 41 (1995) 184. 